Submitted to ApJL

Preprint typeset using $\mathrm{AT}_{\mathrm{E}} \mathrm{X}$ style emulateapj v. 05/04/06

\title{
SPIN ALIGNMENT OF DARK MATTER HALOES IN FILAMENTS AND WALLS
}

\author{
Miguel A. Aragón-Calvo ${ }^{1}$, Rien van de Weygaert ${ }^{1}$, Bernard J. T. Jones ${ }^{1}$ and J.M. (Thijs) van der Hulst ${ }^{1}$ \\ Submitted to ApJL
}

\begin{abstract}
The MMF technique is used to segment the cosmic web as seen in a cosmological N-body simulation into wall-like and filament-like structures. We find that the spins and shapes of dark matter haloes are significantly correlated with each other and with the orientation of their host structures. The shape orientation is such that the halo minor axes tend to lie perpendicular to the host structure, be it a wall or filament. The orientation of the halo spin vector is mass dependent. Low mass haloes in walls and filaments have a tendency to have their spins oriented within the parent structure, while higher mass haloes in filaments have spins that tend to lie perpendicular to the parent structure.

Subject headings: Cosmology: large-scale structure of Universe - Galaxy formation
\end{abstract}

\section{INTRODUCTION}

The origin of the angular momentum of galaxies and their associated dark matter haloes remains one of the most poorly understood subjects in present galaxy formation theories despite its relevance in determining properties such as size and morphological type. According to the Tidal Torque Theory (TTT), galaxies acquire their angular momentum as a consequence of the tidal shear produced by the neighbouring primordial matter distribution (Hovle 1949; Peebles 1969; Doroshkevich 1970; White 1984). A natural consequence of the TTT is a correlation between the angular momentum of haloes and their surrounding matter distribution (Efstathiou \& Jones 1979; Barnes \& Efstathiou 1987; Heavens \& Peacock 1988; Lee \& Pen 2001; Porciani et al. 2002). Lee \& Pen (2001) and Lee (2004) made specific predictions about this.

Orientation studies based on galaxy catalogues show anti-alignment (Kashikawa \& Okamura 1992; Navarro et al. 2004; Truillo et al. 2006). The situation in N-body models is less clear: some dark matter N-body simulations seem not to detect any systemic halo spin alignment (Patiri et al. 2006: Hevmans et al. 2006), while others (Lee \& Pen 2001; Bailin \& Steinmetz 2005; Hatton \& Ninin 2001; Faltenbacher et al. 2002) present evidence that dark matter haloes are aligned with host structures. More recently Altav et al. (2006) found a strong shape alignment of dark haloes in filaments, while Brunino et al. (2006) reported the discovery of a systematic alignment effect in an analysis of dark matter haloes taken from the very large "Millennium" N-body simulation (Springel et al. 2005). We suggest that such ambiguities may in large part be a consequence of the methods used to identify the larger scale structures that host the haloes.

\section{FINDING AND CLASSIFYING STRUCTURE}

Large galaxy surveys such as the $2 \mathrm{dF}$ (Colless et al. 2001) or more recently the Sloan Digital Sky Survey (York et al. 2000) have unambiguously revealed an intricate network of galaxies: the cosmic web. The cosmic web can be described as a mixture of three

\footnotetext{
${ }^{1}$ Kapteyn Astron. Inst., University of Groningen, PO Box 800 9700 AV Groningen, The Netherlands; miguel@astro.rug.nl
}

basic morphologies: clusters which are predominantly spherical, elongated associations of galaxies (filaments) and large planar structures (walls) (Zeldovich 1970; Shandarin \& Zeldovich 1989).

Bond et al. (1996) emphasized that this weblike pattern is shaped by the large scale tidal force fields whose source is the inhomogeneous matter distribution itself (see also van de Wevgaert (2006)). Acccording to TTT the same tidal field also generates the angular momentum of collapsing halos. Thus we would expect the shape and angular momentum of cosmic haloes to be correlated with one another and with the cosmic web elements in which they are embedded.

Revealing such correlations requires the ability to unambigiously identify the structural features of the cosmic web. Several methods have in the past been used in an attempt to identify and extract the morphological components of the observed galaxy distribution (Barrow et al. 1985; Babul \& Starkman 1992; Luo \& Vishniad 1995; Stoica et al. 2005; Colberg et al. 2005; Pimblet 2005) with varying degrees of success. The results presented in this letter are based on a new method, the Multiscale Morphology Filter: "MMF" (Aragón-Calvo et al. 2006). MMF allows us to objectively segment the cosmic web into its three basic morphological components by analysing the properties of the matter distribution hierarchically. With this morphological characterisation we can isolate specific host environments (filaments and walls) for haloes and test predictions from the TTT in a systematic way.

The MMF method is a significant advance on other similar studies of $\mathrm{N}$-body models. $\mathrm{MMF}$ is a technique that locates and classifies various structures by exploiting localised properties of the inertia tensor of the matter distribution on a hierarchy of scales. Since the inertia tensor is directly related to the dynamical forces that drive the tidal torques, MMF is particularly suited for this investigation. The significance of the effects reported here is strong, despite the relatively small size of the N-body simulation, because of the clear cut MMF environmental descriptor.

\section{N-BODY SIMULATIONS AND STRUCTURE}

We ran a cosmological N-body simulation containing $512^{3}$ equal mass dark matter particles inside a cubic box 

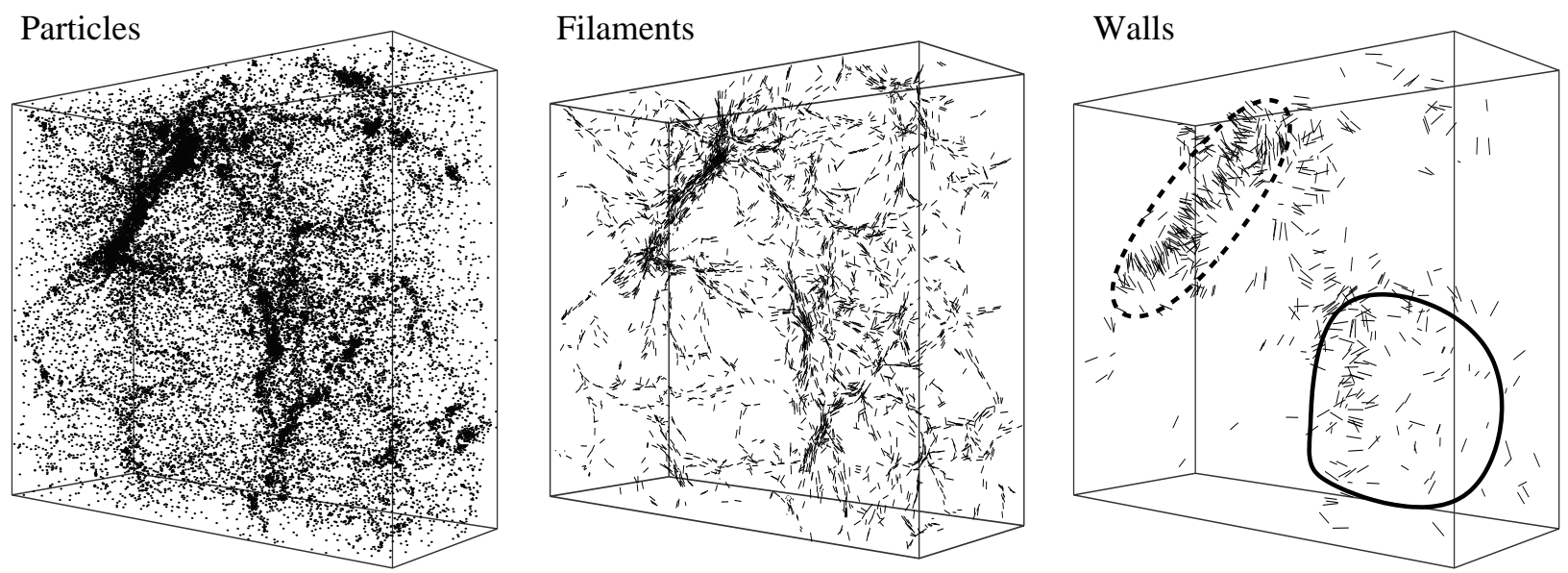

FIG. 1. - Left panel: Particles inside a sub-box of $37.5 \times 75 \times 100 \mathrm{~h}^{-1} \mathrm{Mpc}$. For reasons of clarity only a small fraction of the total number of particles is shown. Central panel: filaments delineated by a subsample of the particle distribution. At each particle location we have plotted the filament vector $\mathbf{e}_{\mathbf{F}}$, indicating the direction locally parallel to the filament. Righthand panel: wall particles detected in the same sub-box: at each wall particle we plot the wall vector $\mathbf{e}_{\mathbf{w}}$. Two walls can be clearly delineated: one seen edge-on (dashed outline) and one seen face-on (solid outline).

of $150 \mathrm{~h}^{-1}$ Mpc. using the public version of the parallel Tree-PM code Gadget2 (Springel 2005). We adopted the standard cosmological model $\Omega_{m}=0.3, \Omega_{\Lambda}=0.7, h=$ 0.7 and $\sigma_{8}=1$. The analysis presented here is based on the last snapshot at $z=0$. The mass per particle is $2 \times 10^{9} \mathrm{~h}^{-1} \mathrm{M}_{\odot}$ and the softening length was set to 18 $\mathrm{h}^{-1} \mathrm{kpc}$ (comoving) until $z=2$ and $6 \mathrm{~h}^{-1} \mathrm{kpc}$ (physical) after that time.

\subsection{Detecting Filaments and Walls}

The Multiscale Morphology Filter is based on the second-order local variations of the density field as encoded in the Hessian matrix $\left(\partial^{2} \rho / \partial x_{i} \partial x_{j}\right)$ for the smoothed density field. For a given set of smoothing scales we compute the eigenvalues of the Hessian matrix at each position on the density field. The density field is computed from the particle distribution by means of the DTFE method (Schaap \& van de Wevgaert 2000, 2006): this is natural and self-adaptive, and retains the intricate anisotropic and hierarchical features characteristic of the cosmic web. DTFE smoothing plays an essential role in delineating filaments and walls unambiguously. We use a set of morphology filters based on relations between the eigenvalues in order to get a measure of local spherical symmetry, filamentariness or planarity. The morphological segmentation is performed in order of increasing degrees of freedom in the eigenvalues for each morphology (i.e. blobs $\rightarrow$ filaments $\rightarrow$ walls). The response from the morphology filters computed at all scales is integrated into a single multiscale response which encodes the morphological information present in the density field. At each stage of the filtering we discard the particles that have previously been assigned to a structure and compute a new density field.

In a filament, the eigenvectors of the Hessian matrix corresponding to the smallest eigenvalue $\left(\mathbf{e}_{\mathbf{F}}\right)$ indicate the direction of filament. In walls the largest eigenvalue, indicates the perpendicular to the wall $\left(\mathbf{e}_{\mathbf{W}}\right)$. Eigenvectors are computed from a smoothed version of the density field; this avoids small-scale variations in the directions assigned to filaments and walls.

In Figure 1 we show a region of the simulation containing several filaments and two large walls detected using the MMF. The box and its projection were chosen in order to avoid confusion from projection of many structures on top of each other and to show one wall face-on and one edge-on. Filaments are clearly delineated like streaming lines joining large associations of matter. Walls are more difficult to visualise so we also plot their defining eigenvectors. For this projection we can see that the eigenvectors $\left(\mathbf{e}_{\mathbf{W}}\right)$ defining the wall seen head-on (solid line) are pointing towards us, while the ones corresponding to the wall seen edge-on are perpendicular to the line-of-sight (dashed line).

\subsection{Halo Identification}

Haloes were identified with a somewhat different implementation of the publicly available halo finder HOP (Eisenstein \& Hut 1998). First we identify haloes by running hop with the standard parameters and $\delta_{\text {out }}=80$, $\delta_{\text {saddle }}=120$ and $\delta_{\text {peak }}=160$ for regrouping. Each of these haloes is considered a parent candidate which may comprise more than one single subhalo. Next for all particles we compute densities using a Gaussian window with dispersion of $35 \mathrm{~h}^{-1} \mathrm{kpc}$, in order to produce a smoothed density field without substructure smaller than this kernel. We run hop again but only for particles inside the parent candidates and this time we also provide the smoothed densities previously computed as an input for hop. The halo identification is performed without running regroup. By doing this hop will assign all particles to their smoothed local maximum, each group found in this stage is a candidate subhalo. For each of these new subhaloes we find their center of mass iteratively and remove the unbound particles. This "FracHop" method allows us to find bound subhaloes inside larger groups otherwise identified as single virialised objects.

Finally, we keep haloes with more than 50 particles and less than 5000, a mass range of $(1-100) \times 10^{11} \mathrm{~h}^{-1} \mathrm{M}_{\odot}$.

\subsection{Halo properties}

The angular momentum of a halo containing $N$ particles is then defined as:

$$
\mathbf{J}=\sum_{i}^{N} m_{i} \mathbf{r}_{\mathbf{i}} \times\left(\mathbf{v}_{\mathbf{i}}-\overline{\mathbf{v}}\right)
$$



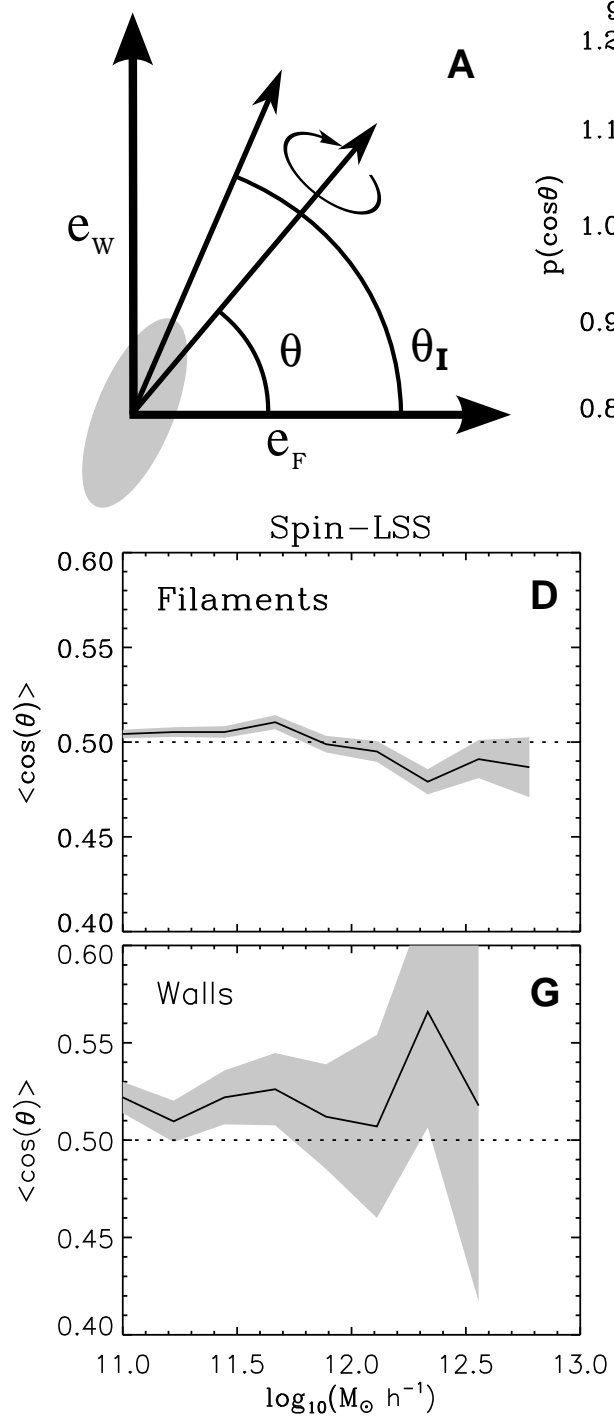

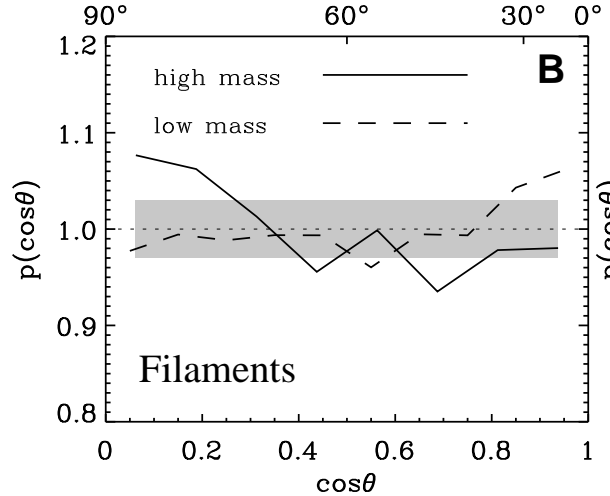

Inertia tensor-LSS

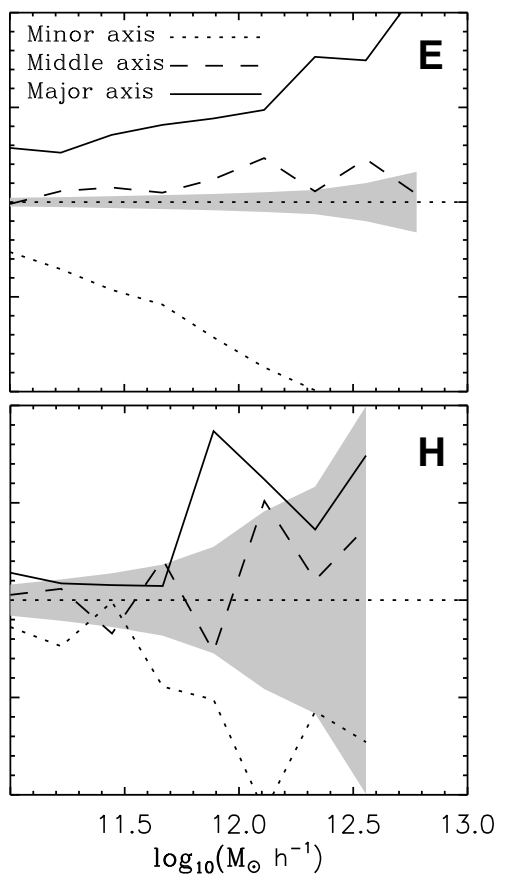

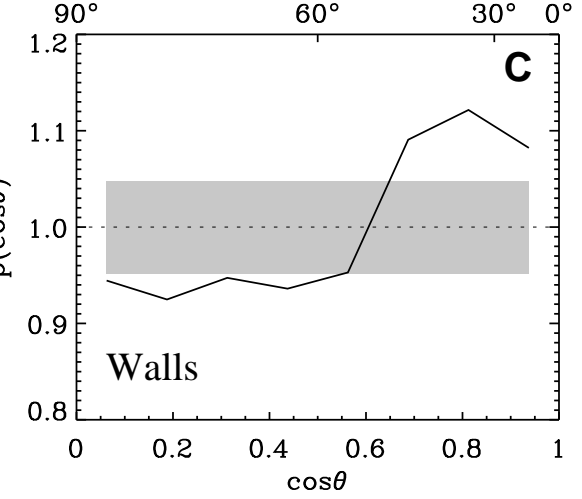

Inertia tensor-Spin
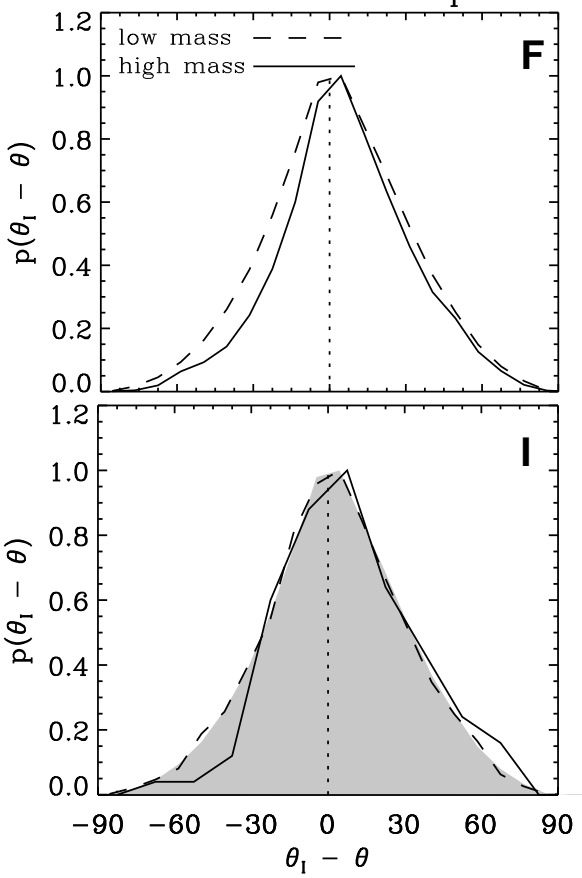

FIG. 2.- A: cartoon illustrating the parameters describing the relative orientations of halos, filaments and walls . B: The probability distribution of $\cos \theta: \theta$ is the angle between the spin vector of a dark matter halos and its host filament. A distinction has been made between haloes with masses smaller and larger than $10^{12} \mathrm{~h}^{-1} \mathrm{M}_{\odot}$ (dashed vs. solid line). The dotted line indicates a uniform distribution of halo orientations. The shaded area corresponds to the standard deviation of 1000 random realisations with the same number of galaxies as the halo sample. The same prescription is followed to estimate and indicate the standard deviation in the other frames. C: Similar to $\mathrm{B}$, for walls: the pdf for the halo spin orientation $\theta$ wrt. the embedding wall. D: The angle $\cos \theta$ between the halo spin direction and the host filament as a function of halo mass $M$. The solid line indicates the average $\langle\cos \theta\rangle$ for the halo sample. The dotted line is the expected distribution for a randomly oriented sample. G: Similar to D, for walls. Note that the shaded deviation band is wider as a result of the lower number of wall haloes. E: The alignment between halo shape and host filament: the average orientation angle $\langle\cos \theta\rangle$ of major (solid line), medium (dashed line) and minor (dotted line) halo axes relative to the filament. H: Similar to E, for walls. F: The pdf of the angle between halo minor axis and halo spin axis for haloes in filaments. The figure distinguishes between low mass (dashed) and high mass haloes (solid). I: The pdf of the angle between halo minor axis and halo spin axis, for haloes in walls. Superimposed (shaded area) is the same distribution for low mass haloes in filaments taken from $\mathrm{F}$.

where $m_{i}$ is the particle's mass, $\mathbf{r}_{\mathbf{i}}$ is the distance of each particle from the center of the halo, $\mathbf{v}_{\mathbf{i}}$ is the peculiar velocity of the particle and $\overline{\mathbf{v}}$ the mean velocity of the halo with respect to the center of mass. We compute the angle between the halo's spin and its assigned Filament or Wall,

$$
\theta_{W}=90-\phi_{W}, \quad \theta_{F}=\phi_{F},
$$

where

$$
\phi_{F, W}=\cos ^{-1}\left(\frac{\mathbf{J} \cdot \mathbf{e}_{\mathbf{F}, \mathbf{W}}}{\left|\mathbf{J} \| \mathbf{e}_{\mathbf{F}, \mathbf{W}}\right|}\right) \text {, }
$$

$\mathbf{e}_{\mathbf{F}, \mathbf{W}}$ being the vector defining the orientation of the filaments (F) and walls (W). For each halo principal axes are computed by diagonalising the inertia moment tensor

$$
\mathbf{I}_{i j}=\sum_{i}^{N} m_{i} x_{i} x_{j}
$$

where the positions of the particles are with respect to the center of mass and the sum is over all particles in the halo. The orientation of any of the eigenvectors of $\mathbf{I}_{i j}$ relative to the host structure is described by angles analogous to the angles $\phi, \theta_{W}$, and $\theta_{F}$ defined above.

\section{RESULTS}

Our main results are presented in Figure 2 and may be summarised as follows: 
- The orientations of halo spins are significantly correlated with the large scale structure in which they are situated. Haloes in walls have spin vectors that tend to lie in the plane of the host wall (frame C). The strength of the alignment of haloes in walls is relatively small (the Lee-Pen correlation parameter is $c=0.13 \pm 0.02$ ) but nevertheless statistically significant: the K-S probability that the halo orientations in walls are randomly distributed is less than $8.16 \times 10^{-5}$. We find similar correlations in filaments, though of a more subtle character (frame B).

- The orientation of halo spin vectors in filaments is mass dependent: low mass halo spins tend to lie parallel to their host filament while high mass halo spins tend to lie perpendicular to their host filament (frames B \& D). High mass haloes, $M>$ $10^{12} h^{-1} M_{\odot}$, have a K-S probability less than $5.50 \times$ $10^{-4}$ of being randomly orientated, low mass haloes less than $1.86 \times 10^{-5}$ (frame B).

- The principal axes of haloes in filaments are strongly correlated with the direction of the filaments: the minor axis tends to be directed perpendicular to the filament, the effect being strongest for the larger masses (frame E)

- The principal axes of haloes in walls are strongly correlated with the perpendicular to walls: the minor axis tends to be lie perpendicular to the wall while the other axes tend to lie in the local plane of the wall (frame $\mathrm{H}$ ).

- While both the halo minor axis and the spin are correlated with the megaparsec scale structure, the distribution of the angle between these two is skewed and biased towards the surrounding large scale structure. This slight skewness may bias the estimate of the mean angle the spin makes with the host structure (frames F and I).

\section{CONCLUDING REMARKS}

The significance of the alignments we have found, given the relatively small sample of haloes and small simulation volume, emphasises the benefits of having a good definition of environmental structures when studying halo properties and their relation with the cosmic web.

The results reported here are in accord with results reported by e.g. Brunino et al. (2006). We find that halo spin vectors lie within the host structure and halo minor axes point out of the host structure as previously suggested by (Patiri et al. 2006; Brunino et al. 2006). Thé major advance of our study is the objective and multiscale character of the structure identification by means of the MMF. Earlier studies at best resorted to heuristic means of delineating filaments or walls (Pimblet 2005; Colberg et al. 2005; Brunino et al. 2006). Some earlier studies have measured the distance of a halo from a local minimum in the large scale density distribution. On the assumption that the surfaces of such "spherical" voids are to be identified with the large scale structures hosting the haloes one may attempt to find signatures of spin and shape alignments. Voids are not generally spherical, nor is it always clear which of several voids a halo is related to. Moreover, using voids as defining the environment of a halo does not in itself allow for the important distinction between walls and filaments. Alignment detections should therefore be seen as residuals of genuine physical alignments with walls or filaments.

This letter poses a number questions for future investigation. The mass segregation of alignments in filaments is yet to be understood. Detailed merging trees may give clues to the possible non-linear processes responsible for this effect. It will of course be interesting to use MMF to define environments in catalogues of galaxies such as $2 \mathrm{dF}$ and SSDS: this should provide an important refinement of earlier results of Truiillo et al. (2006) and since it will unambiguously localise galaxies as either being in walls or in filaments.

We thank Pablo Araya for the N-body simulations used in this work and gratefully acknowledge discussions with Ignacio Trujillo during the "Bernard60" conference in Valencia.

\section{REFERENCES}

Altay G., Colberg J. M., Croft R. A. C. 2006, MNRAS, 370, 1422 Aragón-Calvo M.A., van de Weygaert R., Jones B.J.T., van der Hulst J.M., 2006, to be subm.

Babul A., Starkman G. D. 1992, ApJ, 401, 28

Bailin J., Steinmetz M. 2005, ApJ, 627, 647

Barnes J., Efstathiou G. 1987, ApJ, 319, 575

Barrow J. D., Bhavsar S. P., Sonoda D. H. 1985, MNRAS, 216, 17

Bond J. R., Kofman L., Pogosyan D. 1996, Nature, 380, 603

Brunino R., Trujillo I., Pearce F., Thomas P.A., 2006 , astro-ph/0609629

Chen D. N., Jing Y. P., Yoshikaw K. 2003, ApJ, 597, 35

Colberg J. M., Krughoff K. S., Connolly A. J. 2005, MNRAS, 359 , 272

Colless M., et al. 2001, MNRAS, 328, 1039

Doroshkevich A. G. 1970, Astrophysics, 6, 320

Efstathiou G., Jones B. J. T. 1979, MNRAS, 186, 133

Eisenstein D. J., Hut P. 1998, ApJ, 498, 137

Faltenbacher A., Gottloeber S., Kerscher M., Müller V., 2002 A\&A, 395, 1

Hatton S., Ninin S. 2001, MNRAS, 322, 576

Heavens A., Peacock J., 1988, MNRAS, 232, 339

Heymans C., et al. 2006. astro-ph/0604001

Hoyle F., 1949, Problems of Cosmical Aerodynamics
Kashikawa N., Okamura S. 1992, PASJ, 44, 493

Lee J., Pen U.-L. 2001, ApJ, 555, 106

Lee J. 2004, ApJ, 614, L1

Luo S., Vishniac E. 1995, ApJS, 96, 429

Navarro J. F., Abadi M. G., Steinmetz M. 2004, ApJ, 613, L41

Patiri S. G., et al. 2006. astro-ph/0606415

Peebles P. J. E. 1969, ApJ, 155, 393

Pimblet K.A., 2005, MNRAS, 358, 256

Porciani C., Dekel A., Hoffman Y. 2002, MNRAS, 332, 325

Schaap W. E., van de Weygaert R. 2000, A\&A, 363, L29

Schaap W. E., van de Weygaert R. 2006, A\&A, subm.

Shandarin S.F., Zel'dovich Ya. B., 1989, Revs. Mod. Phys., 61, 185

Springel V. 2005, MNRAS, 364, 1105

Springel V., et al., 2005, Nature, 435, 629

Stoica R., Martínez V., Mateu J., Saar E. 2005, A\&A, 434, 423

Trujillo I., Carretero C., Patiri S. G. 2006, ApJ, 640, L111

van den Bosch F. C., Abel T., Croft R. A. C., Hernquist L., White S. D. M. 2002, ApJ, 576, 21

van de Weygaert R., 2006, in Measuring th diffuse intergalactic medium, eds. J.W. den Herder \& N. Yamasaki

White S. D. M. 1984, ApJ, 286, 38

York D. G., et al. 2000, AJ, 120, 1579

Zel'dovich Ya. B., 1970, A\&A, 5, 84 